\title{
The New "Normal" Blood Pressure: What Are the Implications for Family Medicine?
}

\author{
Anthony J. Viera, MD, MPH
}

Background: In 2003, the Joint National Committee on Prevention, Detection, Evaluation, and Treatment of High Blood Pressure redefined normal blood pressure (BP) as less than 120/80 $\mathrm{mm} \mathrm{Hg}$ and added the category of prehypertension, recommending that such patients receive counseling on lifestyle modifications. Based on population data, $\mathbf{4 1 . 8 \%}$ of US adults have normal BP, $31 \%$ have prehypertension, and the remainder has hypertension. These percentages may not reflect the proportions seen in family medicine practices. The purposes of this study were to describe the proportion of adults in a family medicine practice with normal BP and the proportions with prehypertension and hypertension and to examine associations with having normal BP.

Methods: Records of 633 nonpregnant adults from a large family medicine clinic were reviewed for demographic and BP information. Proportions of subjects in each BP category (normal, prehypertension, or hypertension) were determined. Characteristics associated with normal BP were examined using $\chi^{2}$ tests and logistic regression.

Results: Nearly $80 \%$ of adults in this population had prehypertension or hypertension. Subjects more likely to have normal BP were young, female, white, and not overweight/obese. Almost $60 \%$ of subjects had documented hypertension, were receiving antihypertensive medications, or had a BP on the day of visit $\geq 140 / 90 \mathrm{~mm}$ Hg. Over $20 \%$ had prehypertension.

Conclusion: The proportion of adult family medicine patients with normal BP is low. Counseling $20 \%$ of adult patients about prehypertension while continuing to strive to improve BP control for the $60 \%$ of patients with hypertension could pose a new challenge to clinicians working in family medicine offices. (J Am Board Fam Med 2007;20:45-51.)

The nature of a clinician's work is to collect information so that a decision can be made regarding a course of action to improve a person's health. Such work often dictates that complex information be viewed in a way that can contribute to a decision that something is either normal or abnormal. In medicine, this notion often means that data based on a continuous scale must be dichotomized. In current practice, such has traditionally been the

This article was externally peer reviewed.

Submitted 25 May 2006; revised 1 August 2006; accepted 14 August 2006.

From the Department of Family Medicine, University of North Carolina at Chapel Hill, Chapel Hill, NC.

Financial support: This work was conducted while AJV was a Robert Wood Johnson Clinical Scholar at the University of North Carolina at Chapel Hill.

Conflicts of interest: none declared.

Corresponding author: Anthony J. Viera, MD, MPH, Department of Family Medicine, William B. Aycock Building, CB 7595, Manning Drive, University of North Carolina at Chapel Hill, Chapel Hill, NC 27599 (E-mail: anthony_viera@med.unc.edu). case with blood pressure (BP). Through a series of repeat $\mathrm{BP}$ measurements over time, a clinician makes a decision whether or not to make a diagnosis of hypertension. This decision is generally based on a threshold above that $\mathrm{BP}$ - taking into account various comorbidities - considered elevated enough to justify treatment with medications to reduce cardiovascular disease (CVD) risk. For most patients, this threshold is sustained office BPs greater than 140 $\mathrm{mm} \mathrm{Hg}$ systolic or $90 \mathrm{~mm} \mathrm{Hg}$ diastolic.

In 2003, the Seventh Report of the Joint $\mathrm{Na}$ tional Committee on Prevention, Detection, Evaluation, and Treatment of High Blood Pressure (JNC 7) defined a new category of BP called prehypertension. ${ }^{1}$ The prehypertension category includes nonhypertensive adults with BP between 120 to $139 \mathrm{~mm} \mathrm{Hg}$ systolic or 80 to $89 \mathrm{~mm} \mathrm{Hg}$ diastolic. JNC 7 states that such patients “... require health-promoting lifestyle modifications to prevent CVD." ${ }^{1}$ These lifestyle modifications include weight loss (if overweight); increased physical 
activity; increased intake of whole grains, fruits, and vegetables; and moderation of alcohol intake. Such lifestyle modifications are of course advisable for all individuals regardless of BP status. The assumption is that patients with prehypertension would receive more intensive, or targeted, advice. The previous JNC 6 report, published in 1997, defined the range of BP 130 to $139 \mathrm{~mm} \mathrm{Hg}$ systolic or 85 to 89 $\mathrm{mm} \mathrm{Hg}$ diastolic as high-normal. ${ }^{2}$ BPs below this range, including those 120 to $129 \mathrm{~mm} \mathrm{Hg}$ systolic and 80 to $84 \mathrm{~mm} \mathrm{Hg}$ diastolic, were considered normal. JNC 7 has in effect, expanded the number of individuals considered abnormal.

Normal BP is now defined as systolic BP less than $120 \mathrm{~mm} \mathrm{Hg}$ and a diastolic BP less than 80 $\mathrm{mm} \mathrm{Hg} .{ }^{1}$ A recent population-based study demonstrated that $41.8 \%$ of US adults have normal BP. ${ }^{3}$ However, this proportion may not reflect the proportion of patients with normal BP in the family medicine office. The purposes of this study were to estimate (1) the proportion of adults in a family medicine practice who actually have normal BP and describe characteristics of such patients; and (2) the proportions of adult patients in a family medicine practice who have prehypertension and hypertension.

\section{Methods}

\section{Setting and Population}

The University of North Carolina at Chapel Hill Family Medicine Center is a stand-alone facility that provides comprehensive primary care to a wide variety of patients. Over 20 board-certified family physicians as well as 24 family medicine residents and 3 nurse practitioners regularly see patients in this clinic. Based on administrative data, over 200 patients of all ages are seen per day; $65 \%$ are female. Approximately $30 \%$ are identified as African American and 2\% as Hispanic. Twelve percent are insured by Medicaid and $23 \%$ by Medicare. Among all patient visits, benign essential hypertension is the most frequently coded diagnosis. As is customary in family medicine offices, patients presenting for a clinic visit undergo a set of vital signs measurements (including BP) after their check-in but before their visit with their health care provider. Patients are seated and positioned as recommended by JNC $7 .{ }^{1}$ Measurements are then taken using an automatic oscillometric device and recorded in the electronic medical record by medical assistants, nursing assistants, licensed practical nurses or registered nurses.

\section{Sample}

Using $40 \%$ as the proportion of subjects expected to have normal BP, 639 subjects would allow estimation of the outcome in the study population with a $99 \%$ CI that is \pm 5 , based on the following formula: $N=4 z_{\alpha}{ }^{2} P(1-P) / W^{2}$, where $N$ represents the total subjects required, $z_{\alpha}$ is the standard normal deviate, $P$ represents the expected proportion, and $W$ the width of the CI. ${ }^{4}$

To derive a sample of this size, one clinic day from each of the 5 months preceding the study was randomly selected. Clinic days could have been any day of the work week (Monday through Friday) excluding holidays during the month. Using the university's electronic medical record (EMR) system, the complete list of patients seen on each of the 5 days was generated and printed. The EMR was then used to sequentially review the demographics of each potential subject followed by their vital signs notation, problem list, medication list, and clinic note (if needed) to extract data. Individuals younger than 18 years or pregnant were excluded. Repeat visits for the same individual if he/ she happened to visit the clinic on more than one of the 5 selected days were also excluded.

\section{Variables}

Age was divided into 4 categories. Body mass index (BMI) was calculated from weight and height data and divided into 3 categories corresponding to normal BMI $\left(<25 \mathrm{~kg} / \mathrm{m}^{2}\right)$, overweight (25 to $30 \mathrm{~kg} /$ $\left.\mathrm{m}^{2}\right)$, and obese $\left(\geq 30 \mathrm{~kg} / \mathrm{m}^{2}\right)$. Race/ethnicity information was based on the information contained in the demographic information of the EMR. This information was entered by administrative personnel at the time of patient registration. It could have been obtained by self-report or, if in person, based on the perception of the clerk entering the information. Because of difficulty in obtaining, and poor reliability of, information on smoking status from the EMR, this variable was not included. Hypertension or diabetes mellitus was considered present if documented on the problem list. Problems in this list may be entered manually by clinicians at the time of care, but are also automatically supplemented from billing diagnoses after every visit. A subject was considered to be on antihypertensive medications if any antihypertensive medication was documented on the medication list.

A normal BP measurement was defined as systolic BP less than $120 \mathrm{~mm} \mathrm{Hg}$ and diastolic BP less 
than $80 \mathrm{~mm} \mathrm{Hg}$. High BP measurement was defined as either systolic greater than or equal to 140 $\mathrm{mm} \mathrm{Hg}$ or diastolic greater than or equal to 90 $\mathrm{mm} \mathrm{Hg}$. These were based on the single measurement noted in the vital signs notation for the day of the visit. If no BP measurement was noted in the vital signs notation, the clinic note was searched. If the clinic note did not contain a BP measurement, the subject was excluded.

\section{Statistical Analysis}

First, basic descriptive statistics, histograms and/or linear trend graphs were used to analyze all variables in the study. Proportions (with 95\% CI) of subjects with 1 of the 3 BP diagnoses (normal, prehypertension, hypertension) and proportions within defined BP measurement ranges (normal, prehypertensive, high) were determined. Then, using normal BP (defined above) as the outcome variable, bivariate analysis consisted of analyzing differences in the proportion of subjects having normal BP across strata of demographic variables using $\chi^{2}$ tests. These analyses were stratified by whether or not subjects had documented hypertension or antihypertensive medication(s). Finally, logistic regression was then used to determine the independent associations with having normal BP. These associations are reported as odds ratios. The statistical software package Stata 8.1 (Stata Corp, College Station, TX) was used for all analyses.

\section{Study Approval}

This study was approved by the Committee on the Protection of the Rights of Human Subjects of the Biomedical Institutional Review Board of the University of North Carolina at Chapel Hill.

\section{Results}

\section{Sample Characteristics}

A total of 633 subjects were included. Most subjects in the sample (Table 1) were 35 years and older $(82 \%)$, female $(63 \%)$, and non-Hispanic white (59\%) Nearly three fourths were overweight or obese. Over $40 \%$ had documented hypertension; $18 \%$ had documented diabetes, and $13 \%$ in total had both hypertension and diabetes documented. Nearly $46 \%$ were taking one or more antihypertensive medications.
Table 1. Sample Characteristics $(n=633)$

\begin{tabular}{|c|c|c|}
\hline Characteristic & $\begin{array}{l}\text { Mean } \\
(\mathrm{SD})\end{array}$ & $\begin{array}{l}\text { Percentage } \\
(95 \% \text { CI })\end{array}$ \\
\hline Systolic BP (mm Hg) & $132(20)$ & \\
\hline Diastolic BP (mm Hg) & $77(11)$ & \\
\hline Age (years) & $50.5(17)$ & \\
\hline \multicolumn{3}{|l|}{ Age group } \\
\hline $18-34$ & & $18.0(15.0-21.0)$ \\
\hline $35-49$ & & $32.5(28.9-36.2)$ \\
\hline $50-64$ & & $27.8(24.3-31.3)$ \\
\hline$\geq 65$ & & $21.6(18.4-24.9)$ \\
\hline \multicolumn{3}{|l|}{ Sex } \\
\hline Female & & $62.6(58.8-66.3)$ \\
\hline Male & & $37.4(33.7-41.2)$ \\
\hline \multicolumn{3}{|l|}{ Race/ethnicity } \\
\hline Black & & $32.9(29.2-36.5)$ \\
\hline White & & $59.1(55.2-62.9)$ \\
\hline Hispanic & & $2.1(0.9-3.2)$ \\
\hline Asian & & $3.0(1.7-4.3)$ \\
\hline Other & & $3.0(1.7-4.3)$ \\
\hline $\mathrm{BMI}\left(\mathrm{kg} / \mathrm{m}^{2}\right)$ & $29.5(8.1)$ & \\
\hline \multicolumn{3}{|l|}{$\mathrm{BMI}\left(\mathrm{kg} / \mathrm{m}^{2}\right)$ category } \\
\hline$<25$ & & $28.9(25.4-32.5)$ \\
\hline $25-30$ & & $30.3(26.7-33.9)$ \\
\hline$\geq 30$ & & $40.8(36.9-44.6)$ \\
\hline \multicolumn{3}{|l|}{ Documented health conditions } \\
\hline Hypertension & & $41.1(37.2-44.9)$ \\
\hline Diabetes & & $18.2(15.2-21.2)$ \\
\hline Hypertension and diabetes & & $13.1(10.5-15.7)$ \\
\hline On BP medication(s) & & $45.7(41.8-49.5)$ \\
\hline
\end{tabular}

BMI, body mass index.

\section{Percentage of Subjects Not Normal with Regard to a BP Diagnosis}

When defining the abnormal diagnostic category as including adult patients with previously documented hypertension, those on antihypertensive medication(s), or those with a measurement on the day of visit in the prehypertensive or hypertensive range, only $20.9 \%$ (95\% CI, 17.7-24.0\%) of those in this sample had normal BP (Table 2). Over 58\% of all adult patients had a diagnosis of hypertension, were receiving antihypertensive medication(s), or had a $\mathrm{BP}$ in the hypertensive range $(\geq 140 / 90 \mathrm{~mm} \mathrm{Hg})$ on the day of their visit. Over one fifth $(20.5 \%$, 95\% CI, $17.4 \%-23.7 \%$ ) had prehypertension.

\section{Percentage of Subjects in Each BP Range}

Among the entire sample $(\mathrm{n}=633), 71.4 \%(95 \%$ CI, $67.9-74.9 \%$ ) had a BP measurement on the day of visit that was either in the prehypertensive or 
Table 2. Percentage (95\% CI) of Patients in Each BP Diagnosis Category $(n=633)$

\begin{tabular}{lc}
\hline BP Diagnosis & Percentage (95\% CI) \\
\hline Normal* $^{*}$ & $20.9(17.7-24.0)$ \\
Prehypertension $\dagger$ & $20.5(17.4-23.7)$ \\
Hypertension $\ddagger$ & $58.6(54.8-62.5)$ \\
\hline
\end{tabular}

* No documentation of hypertension diagnosis or antihypertensive medications and normal BP on day of visit.

$\uparrow$ No documentation of hypertension diagnosis or antihypertensive medications and BP 120 to $139 \mathrm{~mm} \mathrm{Hg}$ systolic or 80 to 89 $\mathrm{mm} \mathrm{Hg}$ diastolic on day of visit (with neither in the hypertensive range).

$\ddagger$ Documentation of hypertension diagnosis or antihypertensive medications or BP on day of visit $\geq 140 \mathrm{~mm} \mathrm{Hg}$ systolic or $\geq 90$ $\mathrm{mm} \mathrm{Hg}$ diastolic.

hypertensive range (Table 3). Just over one quarter (28.6\%, 95\% CI 25.1-32.1\%) had a normal BP measurement, that is, a BP less than $120 / 80$ $\mathrm{mm}$ Hg. Among subjects with no documented diagnosis of hypertension or antihypertensive medications, $19.4 \%$ (95\% CI, 15.1-23.7\%) had a high (hypertensive) BP measurement, and another 40\% (95\% CI, 34.6-45.4\%) had a measurement in the prehypertensive range. Thus, only $40.6 \%$ (95\% CI, $35.2-46.0 \%)$ of those without documented hypertension or pharmacologically influenced BP had a normal BP measurement. Among subjects with documented hypertension or on one or more antihypertensive medications, $15.9 \%$ had a normal BP. Approximately one third (32.1\%, 95\% CI, 26.9$37.4 \%$ ) have presumably been treated to the prehypertensive range, and over half $(52.0 \%, 95 \% \mathrm{CI}$, $46.3-57.6 \%$ ) seem to not be at goal BP. That is, their $\mathrm{BP}$ is still in the hypertensive range.

\section{Associations with Having a Normal BP}

In bivariate analyses (Table 4) among subjects with no documented diagnosis of hypertension or antihypertensive medications, younger age, female sex, white race, and lower BMI were associated with having a normal BP. Only $30 \%$ of those 65 years and older had a normal BP, compared with $52 \%$ of those 18 to 34 years of age. Half of females had a normal BP, compared with only 1 of 4 males. Similarly, $43 \%$ of white patients had a normal BP compared with $28 \%$ of black patients. Only one third of overweight/obese patients had a normal $\mathrm{BP}$, compared with half of those with BMI less than $25 \mathrm{~kg} / \mathrm{m}^{2}$. When adjusting for the demographic characteristics as well as BMI, hypertension diagnosis, and antihypertensive medications (Table 5), the associations with younger age, female sex, white race, and lower BMI remained. The strongest factors independently associated with a normal BP were young age (OR 3.2, 95\% CI 1.6 to 6.5 , for age 18 to 34 ) and normal BMI (OR 1.9, 95\% CI 1.2 to 3.0, for BMI <25).

Among subjects with documented hypertension or on one or more anti-hypertensive medications, there were no statistically significant differences in the proportion having a normal $\mathrm{BP}$ based on the characteristics included in the study (Table 4).

\section{Discussion}

In terms of BP measurement, the vast majority of adult patients seen in this family medicine population were not normal. In fact, on a given day, 3 to 4 of every 5 adult patients had a BP measurement in either the prehypertensive or hypertensive range. In terms of diagnoses, $79.1 \%$ of all adult family medicine patients in this sample had either prehypertension or hypertension. This represents an approximately $21 \%$ higher proportion than that seen in the general population of US adults based on 1999 to 2000 National Health and Nutrition Examination Survey (NHANES) data. ${ }^{3}$ This difference probably reflects, at least in part, the higher proportion of illness expected in a clinic population, compared with the general population.

Table 3. Percentage (95\% CI) of Patients in Each BP Measurement Range on Day of Visit, Stratified by Hypertension Diagnosis/Treatment Status

\begin{tabular}{lccc}
\hline BP Measurement & $\begin{array}{c}\text { Entire Sample } \\
(\mathrm{n}=633)\end{array}$ & $\begin{array}{c}\text { No Diagnosis of Hypertension or } \\
\text { Antihypertensive Medications } \\
(\mathrm{n}=325)\end{array}$ & $\begin{array}{c}\text { Diagnosed with Hypertension or } \\
\text { on Antihypertensive Medications } \\
(\mathrm{n}=308)\end{array}$ \\
\hline $\begin{array}{l}\text { Normal }(<120 / 80 \mathrm{~mm} \mathrm{Hg}) \\
\begin{array}{l}\text { Prehypertensive }(120 \text { to } 139 / 80 \\
\text { to } 89 \mathrm{~mm} \mathrm{Hg})\end{array}\end{array}$ & $28.6(25.1-32.1)$ & $40.6(35.2-46.0)$ & $15.9(11.8-20.0)$ \\
$\begin{array}{l}\text { Hypertensive }(\geq 140 / 90 \mathrm{~mm} \mathrm{Hg}) \\
\text { Hy }\end{array}$ & $35.2(32.4-39.9)$ & $40.0(34.6-45.4)$ & $32.1(26.9-37.4)$ \\
\end{tabular}




\begin{tabular}{|c|c|c|c|c|c|c|}
\hline \multirow[b]{2}{*}{ Characteristic } & \multicolumn{3}{|c|}{$\begin{array}{c}\text { No Diagnosis of Hypertension or } \\
\text { Antihypertensive Medications }(\mathrm{n}=325)\end{array}$} & \multicolumn{3}{|c|}{$\begin{array}{c}\text { Diagnosed with Hypertension or on } \\
\text { Antihypertensive Medications }(\mathrm{n}=308)\end{array}$} \\
\hline & $\mathrm{N}$ & Percentage & $P$ Value & $\mathrm{N}$ & Percentage & $P$ Value \\
\hline \multicolumn{7}{|l|}{ Age } \\
\hline $18-34$ & 94 & 52.1 & .002 & 20 & 25.0 & .233 \\
\hline $35-49$ & 142 & 43.0 & & 64 & 15.6 & \\
\hline $50-64$ & 62 & 22.6 & & 114 & 19.3 & \\
\hline$\geq 65$ & 27 & 29.6 & & 110 & 10.9 & \\
\hline \multicolumn{7}{|l|}{ Sex } \\
\hline Female & 203 & 49.8 & $<.001$ & 193 & 14.0 & .233 \\
\hline Male & 122 & 25.4 & & 115 & 19.1 & \\
\hline \multicolumn{7}{|l|}{ Race/ethnicity } \\
\hline White & 203 & 43.4 & .056 & 171 & 19.3 & .399 \\
\hline Hispanic & 12 & 33.3 & & 1 & - & \\
\hline Black & 78 & 28.2 & & 130 & 12.3 & \\
\hline \multicolumn{7}{|l|}{ BMI } \\
\hline$<25$ & 125 & 51.2 & .008 & 58 & 20.7 & .54 \\
\hline $25-30$ & 113 & 32.7 & & 79 & 15.2 & \\
\hline$\geq 30$ & 87 & 35.6 & & 171 & 14.6 & \\
\hline
\end{tabular}

${ }^{*} \mathrm{~N}$ represents the total number of individuals within each category; percentage represents the proportion with normal BP as defined as $<120 \mathrm{~mm} \mathrm{Hg}$ systolic and $<80 \mathrm{~mm} \mathrm{Hg}$ diastolic; Values of $P$ are based on $\chi^{2}$ test of difference in proportions within categories. BMI, body mass index.

Among those who had an abnormal measurement, approximately half were in the hypertensive

Table 5. Independent Associations with Normal BP $(\mathrm{n}=633)^{*}$

\begin{tabular}{lcr}
\hline Characteristic & OR & $95 \%$ CI \\
\hline Age & & \\
$\quad 18-34$ & 3.2 & $1.6-6.5$ \\
$35-49$ & 2.4 & $1.3-4.5$ \\
$50-64$ & 1.4 & $0.74-2.6$ \\
$\geq 65$ & Referent & \\
Sex & & \\
Female & 1.6 & $1.1-2.5$ \\
Male & Referent & \\
Race/ethnicity & & \\
White & 1.8 & $1.1-2.8$ \\
Hispanic & 0.72 & $0.15-3.4$ \\
Black & Referent & \\
BMI (kg/m ${ }^{2}$ ) & & \\
$\quad<25$ & 1.9 & $1.2-3.0$ \\
$25-30$ & 1.2 & $0.73-2.0$ \\
$\geq 30$ & Referent & \\
Hypertension diagnosis & 0.38 & $0.19-0.71$ \\
On BP medication(s) & 1.1 & $0.62-2.2$ \\
\hline
\end{tabular}

* Based on logistic regression adjusted for all other characteristics in table. BMI, body mass index. range and half were in the prehypertensive range. When stratified by hypertensive diagnosis and/or medications, almost $60 \%$ of patients in this family medicine clinic population with no prior diagnosis of hypertension or on antihypertensive medications had a BP measurement that was not normal, and $19.4 \%$ may have yet undetected or untreated hypertension (ie, $\mathrm{BP} \geq 140 / 90 \mathrm{~mm} \mathrm{Hg}$ ). However, it is not known who among this $20 \%$ had a repeat measurement in the clinic that was actually less than 140/90 $\mathrm{mm} \mathrm{Hg}$, or who might be undergoing repeat evaluations of their elevated $\mathrm{BP}$ to establish a diagnosis, or who is being evaluated for possible "white-coat" hypertension.

Among patients known (documented) to have hypertension or receiving antihypertensive medications, $52 \%$ are apparently uncontrolled, ie, have a $\mathrm{BP}$ greater than $140 \mathrm{~mm} \mathrm{Hg}$ systolic or greater than $90 \mathrm{~mm} \mathrm{Hg}$ diastolic. Again, this percentage is based solely on one measurement and does not take into account factors such as a repeat measurement by the clinician or possible "white-coat" effect. However, it also does not take into account the possibility of masked hypertension ${ }^{5}$ or that goal BP for some groups (eg, diabetics) is actually even lower. ${ }^{1}$ Although this percentage is less than the 
$69 \%$ of patients in the general US population who do not have their hypertension under $\operatorname{control}^{6}$ and is close to the Healthy People 2010 population goal of $50 \%,{ }^{7}$ it still leaves much room for improvement.

Certainly, it is not common for people 65 years and older to have a $\mathrm{BP}$ in the normal range, and these data affirm this: $30 \%$ of those 65 years and older with no documented hypertension or antihypertensive medications, and only $11 \%$ of those 65 years and older with documented hypertension or antihypertensive medications, had a normal BP.

The findings that younger age and lower BMI are associated with having a normal BP are not surprising. In this study, the bivariate associations with normal BP do not hold true among those who have documented hypertension or antihypertensive medications. This suggests that patients are equally unlikely to be treated to- or it is equally unlikely to achieve-a BP goal that is as aggressive as normal once they have reached the treatment threshold.

As anticipated, many patients' BPs fall within the prehypertensive range. Among patients documented to have hypertension or receiving antihypertensive medications, $32 \%$ had a BP measurement in the prehypertensive range. This is certainly reasonable given that the goal $\mathrm{BP}$ in those at highest cardiovascular risk is still within the prehypertensive range. Among those with no prior diagnosis of hypertension or on antihypertensive medications, $40 \%$ had BPs considered prehypertensive. These are the patients who are recommended to receive counseling regarding lifestyle recommendations to lower their risk of progression to hypertension. This represents a substantial number of family medicine patients. Such counseling also requires a large amount of additional time in an already busy setting, and the outcomes are uncertain. It will remain a challenge for clinicians to develop the skills needed to effectively counsel these at-risk patients, while at the same time performing better case-finding for undiagnosed hypertensives as well as more intensive treatment for those already under partial treatment.

\section{Limitations}

The diagnosis codes used in this study were based on documentation in the EMR. It is possible that a patient could have had a diagnosis of hypertension that was not recorded in the record by the clinician.
However, diagnoses from billing codes are automatically added to this list, so this is likely to be a minor issue. Medication usage was also based on documentation in the EMR. Medications not listed in the EMR would not have been noted. It is also recognized that some antihypertensives are used to treat conditions other than hypertension. However, the effects of these limitations on the estimates are likely to be minimal.

BP measurements in this study were based on the measurement taken by the nurses or medical assistants at the vital signs station. Some of these measurements may have been falsely high (eg, if too small a cuff was used) or falsely low (eg, if too large a cuff was used). However, the means in a large clinic sample are unlikely to be significantly affected. In addition, it is recognized that a diagnosis of prehypertension should be based on at least 2 BP readings from at least 2 separate office visits. ${ }^{1}$

This study was conducted in one large clinic in the Southeastern United States with a primary care population that may not be representative of other clinics or locations. Thus, the results may not be generalizable. This may be particularly true for clinics that have a larger elderly population, relatively few younger adults, or a lower proportion of women. However, such populations would probably have an even higher proportion of patients with abnormal BP. The results also may not be generalizable to clinic populations with a lower proportion of black patients. Estimates of proportions of patients with abnormal BP in such a population might be lower than seen in this study.

\section{Conclusions}

Only one of every 5 nonpregnant, adult patients seen in a family medicine setting has BP that would be considered normal by the JNC 7 definition. Such patients are more likely to be young and not overweight or obese. They are also more likely to be female and white. The remaining $80 \%$ of adult patients have either hypertension or prehypertension. Among patients with hypertension, over half have $\mathrm{BP}$ measurements that are not at goal. Although current guidelines suggest more aggressive approaches (ie, counseling) to those with prehypertension, improving BP control among those who have already crossed the treatment threshold should remain a priority. In terms of effectiveness of clinical interventions, the number of patients 
with established hypertension who need to be treated (ie, number needed to treat) to achieve benefit will be exceedingly lower than the number of patients with prehypertension who need to be counseled to achieve benefit. Given that nearly half of patients with established hypertension may be undertreated, truly expanding the number of patients for whom the clinician should intervene should be done with care. If prehypertension needs to be addressed in the clinical setting, clinicians will probably need to seek innovative ways to effectively counsel such patients, perhaps through group visits or use of allied health care team members. Alternatively, the best method to counsel people about prehypertension might be outside the clinic setting.

The author thanks Timothy Carey, MD, MPH, and Donald Pathman, MD, MPH, for reviewing this manuscript and providing helpful feedback and suggestions.

\section{References}

1. Chobanian AV, Bakris GL, Black HR, et al. The Seventh Report of the Joint National Committee on Prevention, Detection, Evaluation, and Treatment of High Blood Pressure: the JNC 7 report. JAMA 2003;289:2560-72.

2. The Sixth Report of the Joint National Committee on Prevention, Detection, Evaluation, and Treatment of High Blood Pressure. Arch Intern Med 1997;157:2413-46.

3. Wang Y, Wang QJ. The prevalence of prehypertension and hypertension among US adults according to the new joint national committee guidelines: new challenges of the old problem. Arch Intern Med 2004;164:2126-34.

4. Hulley SB, Cummings SR, Browner WS, et al. Designing Clinical Research. 2nd Ed. Philadelphia: Lippincott Williams Wilkins, 2001.

5. Bobrie G, Chatellier G, Genes N, et al. Cardiovascular prognosis of "masked hypertension" detected by blood pressure self-measurement in elderly treated hypertensive patients. JAMA 2004;291: 1342-9.

6. Hajjar I, Kotchen TA. Trends in prevalence, awareness, treatment, and control of hypertension in the United States, 1988-2000. JAMA 2003;290: 199-206.

7. United States Department of Health and Human Services. Healthy People 2010. Washington, DC: US Department of Health and Human Services, 2000. 